\title{
Fine-grained provenance of users' interpretations in a collaborative visualization architecture
}

\section{Citation for published version (APA):}

Al-Naser, A., Rasheed, M., Irving, D., \& Brooke, J. (2014). Fine-grained provenance of users' interpretations in a collaborative visualization architecture. In IVAPP 2014 - Proceedings of the 5th International Conference on Information Visualization Theory and Applications/IVAPP - Proc. Int. Conf. Inf. Vis. Theory Appl. (pp. 305-317). Science and Technology Publications Lda.

\section{Published in:}

IVAPP 2014 - Proceedings of the 5th International Conference on Information Visualization Theory and Applications|IVAPP - Proc. Int. Conf. Inf. Vis. Theory Appl.

\section{Citing this paper}

Please note that where the full-text provided on Manchester Research Explorer is the Author Accepted Manuscript or Proof version this may differ from the final Published version. If citing, it is advised that you check and use the publisher's definitive version.

\section{General rights}

Copyright and moral rights for the publications made accessible in the Research Explorer are retained by the authors and/or other copyright owners and it is a condition of accessing publications that users recognise and abide by the legal requirements associated with these rights.

\section{Takedown policy}

If you believe that this document breaches copyright please refer to the University of Manchester's Takedown Procedures [http://man.ac.uk/04Y6Bo] or contact uml.scholarlycommunications@manchester.ac.uk providing relevant details, so we can investigate your claim.

\section{OPEN ACCESS}




\title{
Fine-Grained Provenance of Users' Interpretations in a Collaborative Visualization Architecture
}

\author{
Aqeel Al-Naser ${ }^{1}$, Masroor Rasheed ${ }^{2}$, Duncan Irving ${ }^{2}$ and John Brooke ${ }^{1}$ \\ ${ }^{1}$ School of Computer Science, The University of Manchester, United Kingdom \\ ${ }^{2}$ Teradata Corp. \\ aqeel.al-naser@cs.manchester.ac.uk,masroor.rasheed@teradata.com,duncan.irving@teradata.com, \\ john.brooke@manchester.ac.uk
}

Keywords: Geospatial Visualization, Data Acquisition and Management, Provenance, Data Exploration, Query-Driven Visualization.

\begin{abstract}
In this paper, we address the interpretation of seismic imaging datasets from the oil and gas industry-a process that requires expert knowledge to identify features of interest. This is a subjective process as it is based on human expertise and thus it often results in multiple views and interpretations of a feature in a collaborative environment. Managing multi-user and multi-version interpretations, combined with version tracking, is challenging; this is supported by a recent survey that we present in this paper. We address this challenge via a data-centric visualization architecture, which combines the storage of the raw data with the storage of the interpretations produced by the visualization of features by multiple user sessions. Our architecture features a fine-grained data-oriented provenance, which is not available in current methods for visual analysis of seismic data. We present case studies that present the use of our system by geoscientists to illustrate its ability to reproduce users' inputs and amendments to the interpretations of others and the ability to retrace the history of changes to a visual feature.
\end{abstract}

\section{INTRODUCTION}

One of the most powerful benefits that visualization brings to data analysis is the ability to harness the intuition of the user in the process of understanding the data. Human visual abilities are particularly tuned to respond to features embedded in three dimensional space. In this paper, we consider seismic imaging data which has a natural representation in the three dimensions of physical space composed of subsurface layers and is rich of geological features such as horizons and faults.

In many cases, human intuition is supported by algorithms that help to identify and highlight features of the data. However, it can often be the case that the algorithms cannot completely identify the features of interest. Human intuition must complete the process, and given the nature of intuition this can be a source of differing interpretations depending on the human expert. This may particularly occur in data that is noisy or visually complex. Examples of such data are found in medical imaging and in the field that is the topic of this paper, interpretation of geophysical seismic imaging data (Robein, 2010). Thus we do not have a single feature, but multiple interpretations of a feature. At some stage, collaborative visualization may be required for experts to discuss and reconcile these different interpretations. We also need to track the provenance of such interpretations; sometimes earlier interpretations may need to be revisited. The process can be envisaged as being similar to the source trees created by different programmers working on a large software project and the version control systems that have arisen to manage the process of collaboration and integration. Conventionally, users' interpretations are stored as geometric objects separately from the data and possibly on different local machines; therefore keeping track of these interpretations becomes very complex and error prone.

In this paper we propose a novel method of creating the objects that underlie such visual interpretations, in such a way that the information contained in the interpretation is directly stored as metadata alongside the original data. In addition to the ability of users to experiment with local views of data, this provides support for the considered results of such experiments to be stored, shared and re-used. In our proposed architecture, users' interpretations can flow 
in the reverse direction from the usual pipeline, back from the user's interaction with the visual presentation of the objects to the original data source of the pipeline. This pipeline features a fine-grained dataoriented provenance, which is not available in current methods for visual analysis of seismic data. We utilize the increased capabilities of highly parallel databases that allow flexible indexing and optimized retrieval in response to data queries. Such databases have been created to solve problems of "big data" from the commercial world such as customer relationship management and inventory and asset control.

In this paper, we apply our methods to the problems of the interpretation of data from geoseismic surveys. This is a field that has received a great deal of attention in terms of research (e.g. (Plate et al., 2002; Castanie et al., 2005; Lin and Hall, 2007; Plate et al., 2007; Patel et al., 2009; Patel et al., 2010; Höllt et al., 2011)) as well as software development such as Avizo Earth (Visualization Sciences Group, 2013), GeoProbe (Halliburton-Landmark, 2013), and Petrel (Schlumberger, 2013). The data in this field is noisy and the features to be extracted have a very complex spatial structure owing to processes of buckling, folding and fracturing (Robein, 2010). This makes a purely automated approach to feature extraction very difficult to achieve. The expert interpretation is very central to the definition of the features and the considerations outlined above are of critical importance (Bacon et al., 2003).

This paper presents a continuation of a work that we have previously published (Al-Naser et al., 2013a; Al-Naser et al., 2013b); here we present further analysis and evaluation. The contributions of this paper are as follows:

1. A visualization architecture which allows capturing multi-user understanding (interpretation) of spatial datasets with a fine-grained data provenance.

2. An evaluation of the proposed architecture by geoscientists.

The structure of the paper is as follows. In Section 2 we review related work. In Section 3 we show the abstract principles of our fine-grained data-centric visualization architecture. In Section 4 we describe how we implement these principles in the field of geoscience. In Section 5 we present the results of our case studies, some performance measure, and a survey we have conducted. Section 6 presents conclusions and plans for future work.

\section{BACKGROUND AND RELATED WORK}

\subsection{The Visualization Pipeline}

The visualization pipeline builds the visual objects presented to the user in the form of a data processing workflow that starts from the original data right to the rendering on the display device. This basic formulation has proved very durable and has undergone extensive elaboration since its formulation for over twenty years (Moreland, 2013). A basic visualization pipeline features the following modules in the order of execution: reader $\rightarrow$ geometry generator $\rightarrow$ renderer. Improvements and elaborations have been proposed to address variety of issues such as visualizing multiple datasets, visualizing large datasets, and enhancing performance and efficiency.

A fuller description of data by metadata enhanced the power of visualization by allowing a more fullfeatured view of the data, taking into account its special properties and allowing users flexibility in creating visual objects. Using metadata, users can select a region or multiple regions to process, for example this allowed Ahrens et al. to visualize largescale datasets using parallel data streaming (Ahrens et al., 2001). In addition to regional information, a time dimension can be added to metadata, adding time control to the visualization pipeline (Biddiscombe et al., 2007). The usefulness of metadata was further developed with the introduction of query-driven visualization (Stockinger et al., 2005; Gosink et al., 2008). Query-driven pipelines require the following technologies: file indexing, a query language and a metadata processing mechanism to pass queries. For fast retrieval, indexing technologies are used, such as FastBit (Wu, 2005; Wu et al., 2009) (based on compressed bitmap indexing) and hashing functions. To handle massive datasets efficiently, the visualization pipeline can be executed in parallel over multiple nodes. This data parallelism can be implemented with a variety of parallel methods, for example by MapReduce (Vo et al., 2011). Database management systems (DBMS) are also capable of parallel execution of analysis but were not widely used for the purpose of visualization. A comparison between MapReduce and DBMS was presented by Pavlo et al. (Pavlo et al., 2009); the authors suggest that DBMS has a performance advantage over MapReduce while the latter is easier to setup and to use. 


\subsection{Data Provenance and Management}

Provenance, or lineage, is metadata that allows retrieval of the history of the data and how it was derived. Simmhan et al. (Simmhan et al., 2005) and Ikeda et al. (Ikeda and Widom, 2009) in their surveys categorize provenance techniques in different characteristics, two of which are (1) subject, or type, of provenance and (2) granularity. Data provenance can be of two types: (1) data-oriented, or wherelineage, which is a provenance applied on the data explicitly answering the question of which dataset(s) contributed to produce the output, and (2) processoriented, or how-lineage, which is a provenance applied on the process answering the question of how the resulting output was produced from the input data. Each of the two types can be applied on one of two granularity levels: (1) coarse-grained (schema level) and (2) fine-grained (instance-level). The latter deals with individual data items separately.

The first software to bring the concept of provenance into visualization was VisTrails (Bavoil et al., 2005; Scheidegger et al., 2007). In VisTrails, the changes to the pipeline and parameter values are captured, allowing to review and compare previous versions. Thus, following the taxonomy of provenance by Simmhan et al. (Simmhan et al., 2005), VisTrails can be classified as a process-oriented model. We share a similar aim in respect to maintaining data provenance, but our method differs in the type of metadata that is being captured from the user's visual exploration. We adopt a data-oriented provenance model by explicitly storing metadata of the data points of the interpreted features. This is due to the nature of the data we deal with in this paper; a process-oriented model is not sufficient in our case as interpreted features are mainly a result of users' visual understanding. This was highlighted in a recent study on the value of data management in the oil and gas industry (Hawtin and Lecore, 2011). The study showed that human expertise contributed by $32.7 \%$ towards understanding the subsurface data for the purpose of interpretation; the other elements were data which contributed by $38 \%$, tools which contributed by $15.1 \%$, and process which contributed by $13.7 \%$. This means that human expertise is essential to interpret the data, with a minor help from some tools (algorithms). Despite such impressive development, the evolution of the visualization pipeline has not (to our knowledge) developed a reverse direction to directly link the users' understanding of the data back into the dataset.

Data management is of a concern for systems with an integrated visualization. MIDAS (Jomier et al.,
2009), a digital archiving system for medical images, adopts a "data-centric" solution for massive dataset storage and computing to solve issues like moving data across networks for sharing and visualization. The visualization of MIDAS was originally provided through its web interface. Later, Jomier et al. (Jomier et al., 2011) integrated ParaViewWeb, a remote rendering service based on ParaView, with MIDAS to directly visualize the centrally located large datasets. In the oil and gas industry, Schlumberger's Studio E\&P (Alvarez et al., 2013), an extension to its commercial software Petrel (Schlumberger, 2013), allows a multiuser collaboration via a central data repository. Users import a dataset to visualize and interpret from Studio $E \& P$ to their local Petrel, then commit their changes back to the central Studio E\&P. These two recent examples, from the fields of medical imaging and oil and gas, show us a trend toward centralizing users' data to allow an efficient collaboration and ease data management. These solutions offer a provenance of a coarse-grained granularity, which deals with individual files as the smallest provenance unit. In this paper, we continue with this direction of a data-centric architecture via further integration into the visualization architecture, based on a fine-grained granularity, applying provenance to tuples in the database.

\subsection{Seismic Imaging Data-The Oil and Gas Industry}

In this paper, we apply our method to seismic imaging data from the oil and gas industry. To acquire seismic data, acoustic waves are artificially generated on the earth's surface and reflect from subsurface geological layers and structures. Due to the variation of material properties, these waves are reflected back to the surface and their amplitudes and travel time are recorded via receivers (Ma and Rokne, 2004). This data is then processed to generate a $2 \mathrm{D}$ or $3 \mathrm{D}$ image illustrating the subsurface layers. A 2D seismic profile consists of multiple vertical traces. Each trace holds the recorded amplitudes sampled at, typically, every four milliseconds. Seismic imaging is then interpreted by a geoscientist to extract geological features such as horizons and faults. This interpretation potentially identifies hydrocarbon traps: oil or gas. In 2011, the consumption of fossil fuels (oil and gas) accounted for $56 \%$ of the world's primary energy as calculated by BP (BP, 2012b). By 2030, it is projected that the demand of fossil fuels will continue to grow (BP, 2012a). Thus, this industry requires to manage and visualize its massive datasets more efficiently.

The SEG-Y format has been used by the industry to store seismic data since mid 1970s. SEG-Y 
structure consists of a textual file header, a binary file header, and multiple trace records. Each trace record consists of a binary trace header and trace data containing multiple sample values; more details can be found in the SEG-Y Data Exchange Format (revision 1) (Society of Exploration Geophysicists, 2002).

Data in a SEG-Y file is stored sequentially and therefore retrieval of seismic data for a $3 \mathrm{D}$ visualization could negatively affect interactivity. For this reason, seismic visualization and interpretation applications, such as Petrel (Schlumberger, 2013), offer an internal format which stores seismic data in multiresolution bricks for fast access; this is based on the Octreemizer technique by Plate et al. (Plate et al., 2002). This has been a successful approach in visualizing very large seismic data. However, data management is still a challenge, mainly in managing multiuser interpretations and moving data between users and applications; this was confirmed to us through discussions with geoscientists from the oil and gas industry and through a survey which we present in Section 5.3. Current seismic applications often use proprietary internal formats and also represent and store interpreted surfaces such as horizons and faults in objects stored separately from the seismic data.

\subsection{Other Related Work}

In order to maintain users' data provenance coherently with the data, we need to create data structures that contain both; thus the original data becomes progressively enhanced as the users visualize it. An architecture needs to be developed that can incorporate this enhancement in a scalable manner. Al-Naser et al. (Al-Naser et al., 2011) were first to introduce the concept of feature-aware parallel queries to a database in order to create a volume in real time ready for direct volume rendering. In this approach, features-which are classically represented by meshes-are stored as points tagged into the database; thus queries are "feature-aware." Their work was inspired by Brooke et al. (Brooke et al., 2007) who discussed the importance of data locality in visualizing large datasets and exploited the (then) recently available programmable GPUs for direct rendering without the creation of geometric objects based on meshing. This definition of "feature-aware" differs from that used by Zhang and Zhao (Zhang and Zhao, 2010) in which an approximation is applied for time-varying mesh-based surfaces to generate multi-resolution animation models while preserving objects' features.

With the rapid advances in the capabilities of GPUs, direct volume rendering techniques such as 3D texture slicing (Mcreynolds and Hui, 1997) and
GPU-based ray casting (Hadwiger et al., 2009) have become more efficient for interactive visualization on a large uniform grid. The latter was inspired by the introduction of shading languages such as OpenGL Shading Language (GLSL). We exploit such standard techniques in our architecture to support the primary claim in this paper; we plan to utilize other advanced techniques in future to deal with different data structures, e.g. unstructured spatial data.

\section{DATA-CENTRIC VISUALIZATION ARCHITECTURE}

In this section, we present our solution at an abstract level; in the next section (4) we discuss its implementation in the field of geoscience for the oil and gas industry in particular. Using standard technologies, we propose a data-centric visualization architecture which stores users' interpretations back to the central database for reusability and knowledge sharing. We choose to build our data structure in a parallel relational database management system (RDBMS); we call this spatially registered data structure (SRDS) (Section 3.1). Since our data structure is stored in a relational database rather than in raw image files and geometric objects, we require an intermediate stage which builds in real time a volume in a format which can be directly rendered on the GPU. We call this a feature-embedded spatial volume (FESVo) (Section 3.2).

As illustrated in Figure 1, the architecture links the SRDS on a database to one or more on-the-fly created FESVo through parallel feature-aware and global spatially-referenced queries which results in a parallel streaming of data units. FESVo, on the other side, is linked to a rendering engine. Users' interpretations are stored back to SRDS. In our current work, we directly store interpretations to SRDS and rebuild the intermediate local volume (FESVo) with the newly added data; in future work we can optimize this by caching users' interpretations in FESVo then later store it into SRDS.

\subsection{Spatially Registered Data Structure (SRDS)}

We follow the direction of a data-centric architecture as in MIDAS (Jomier et al., 2009) (discussed in Section 2.2) to maximize data access by multiple users and thus provide efficient sharing. Our data structure is fine-grained so that each voxel-equivalent data unit 


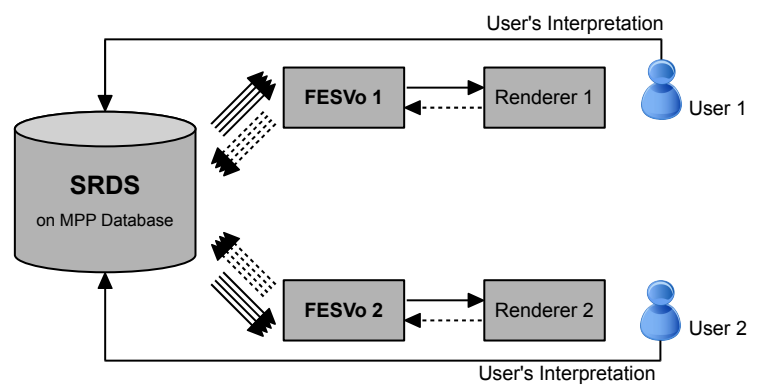

Figure 1: This is a conceptual diagram of a data-centric visual analysis architecture which consists of three loosely coupled components. The (1) spatially registered data structure (SRDS) in a centrally located database is linked to multiple on-the-fly created (2) feature-embedded spatial volumes (FESVo) through parallel connections. FESVo is linked to (3) a renderer engine. Users' interpretations are directly fed back to SRDS; FESVo is then refreshed. Dashed arrows indicate SQL queries from FESVo to SRDS (0.2$0.7 \mathrm{~KB}$ each) or requests for a texture buffer from a renderer to FESVo. Full arrows indicate data units transfer from the database to FESVo (around 4KB each), a texture buffer from FESVo to a renderer (multiple Mbytes) or updates from users to SRDS.

is stored as a tuple, a row in a database. To support querying of tuple-based provenance (Karvounarakis et al., 2010), we restructure our data into a relational form. As a result, our visual analysis method shifts from the classic static raw file system into a central data structure built on a relational database. This also caters for the highly-structured relational modelling required by the integrated analytics paradigm of enterprise-scale business computing.

This structure principally features:

1. global (geographical) spatial reference on all data tuples,

2. interpretation tagging which accumulate users' interpretations into the database,

3. concurrent access allowing parallel multithreading queries from multiple users.

Figure 2 illustrates an abstract database schema of fundamental requirements of SRDS, with no field implementation. Table SRDS holds raw spatial datasets and users' spatial interpretations in a fine-grained structure where each point in a 3D space is stored as a tuple (row). The data is indexed on the combination of $(x, y)$ coordinate and source ID (src_id). A source ID groups data units of one source under a unified identification (ID). The property ID (prop_id) field describes the type of the property value (prop_val) exists at an $(x, y)$ location. For example, a property ID of $\mathbf{1}$ describes a seismic trace type data, a property ID of $\mathbf{2}$ describes a horizon geological feature, and a property ID of 3 describes a fault geological feature. The verti-



Figure 2: This diagram illustrates the abstract SRDS schema. Abbreviations used here are as follows; src_id: source identification; $\mathbf{s r c}$ _ds: source description; ts: timestamp; prop: property; rel_type_cd: relation type code; eff_ts: effective timestamp; end_ts: end timestamp.

cal distance $(\mathrm{z})$ adds a third dimension and timestamp $(t s)$ allows versioning.

Table SRC serves as a source metadata table. It identifies the type of a source (e.g. raw readings, user-interpretation, etc). Table SRC_SRC_GRPING_HIST (source-to-source grouping history) allows data provenance. The table links related interpretations and determines the relation type: e.g. insertion or deletion. The different source ID for each source of an interpretation and the timestamp field make this operation possible.

\subsection{Feature-Embedded Spatial Volume (FESVo)}

Since our data is restructured into a fine-grained form in SRDS, we need an intermediate stage to prepare data for rendering; we call this a feature-embedded spatial volume (FESVo). FESVo's primary roles are to (1) load data, (2) cache data, and (3) capture users' interpretations.

First, FESVo uses the indexing and parallel capabilities of the data structure to perform parallel queries, and an on-the-fly downsampling if a lower resolution is required, resulting in an intermediate volume which can be directly rendered on the GPU. Conventionally, data in 3D spatial volumes is stored in a firmed order and thus it can be read sequentially. However, an SRDS single datum is queried against its geographical location. Therefore, the loading mech- 
anism in FESVo maps between the different coordinate systems: (1) geographical coordinate in SRDS, (2) intermediate volume coordinate in FESVo, and (3) texture coordinate in the GPU. We use a standard rendering technique to visualize this volume which is the data supplied by SRDS format. In Section 4.2, we explain how we implement this process for seismic imaging data.

Second, FESVo also acts as a data cache. The loaded dataset is cached and thus users can fully interact with and interpret it if the connection with SRDS is lost. Third, FESVo captures users' visual interpretations along with provenance metadata. The captured interpretation is mapped back from a local (FESVo) coordinate into a fine-grained geographical coordinate (SRDS).

\section{IMPLEMENTATION FOR GEOSCIENCE}

In this section, we first discuss the extension of SRDS and how data is initially prepared into it. Then, we discuss the continuous loading process by FESVo and how multiple users can contribute and track history. Finally, we briefly describe our rendering engine which renders the data loaded by FESVo.

\subsection{SRDS for Geoscience}

We extend SRDS from its abstract version (Figure 2) to deal with geological and geophysical data. We implement SRDS on a Teradata database; thus we use standard SQL for queries and data updates. We use a hashing index in our tables; this allows an on-the-fly indexing using a hashing algorithm for direct access to data units and efficient update.

Figure 3 illustrates the extended database schema of SRDS for geoscience. We add a dedicated table for seismic traces (raw data), SRDS_TRACE. The main difference between the SRDS and SRDS_TRACE tables is the type of the property value (prop_val) field. The property value field of SRDS_TRACE table is of a customized binary-based type to hold trace samples; this is equivalent to a 1D dataset. Thus, the granularity of SRDS_TRACE table, which is a 1D dataset in a tuple, is coarser than SRDS table, which is a single point datum in a tuple. For SRDS table, the property value (prop_val) field may either represent a single measured value (e.g. porosity, permeability) or an identification of a geological body (e.g. horizon) to this tuple; we adopt the latter at this stage. Thus, we store users' interpretations of geological features as a cloud of points; each point is in a row. Table SRC is extended to fully describe subsurface (seismic) datasets. It determines the boundary of the dataset and range of the amplitude values in the trace sample.

Using a hashing algorithm (Rahimi and Haug, 2010), the location of the required row can be determined through hashing functions without a construction or storage complexity; this is a feature offered by Teradata DBMS. This allows retrieval and writing back from and to the database at a complexity that is proportional only to the working dataset (the size of the dataset being retrieved or written back) and not to the total size of the tables.

\subsubsection{Data Input into SRDS}

In our case, the data is initially prepared from SEG-Y files and geometry as illustrated in Figure 4. We start from post-stack 3D seismic conventional files (SEG$\mathrm{Y}$ format) and extract traces, which are 1D vertical subsurface readings of amplitude values. The trace data is loaded into the database tagged with its geographical location, which is extracted from the trace header. Geological features, which were previously interpreted by users, are obtained in the form of geometry. This is converted into an $(x, y, z)$ cloud of points and loaded into the database. Then, the ongoing users' amendments to the features are directly stored in the same format, as a cloud of points with proper tagging and provenance metadata.

\subsection{Data Loading}

The data loading processes to render a dataset are as follows. A rendering engine requests a ready texture buffer to be directly rendered from FESVo based on a user request of desired datasets. Inside FESVo, a data loader calculates FESVo's current dimension and performs coordinate mapping (described in Section 4.2.1) between SRDS, FESVo and the GPU texture buffer. Upon the user's request, the data loader calculates the fine-grained data units (tuples) required to build the texture buffer. For each data unit, it first checks its internal cache. The data unit, if found, is placed in the texture buffer at the computed (mapped) position. If a data unit is, otherwise, not cached, the unit is added to one of a number of queues in a load balancing manner. Each queue is associated with a thread. After completing the search in the internal cache of FESVo, the threads start, each with its queue of data units (locations) to be concurrently fetched from the database. Each fetched data unit is loaded to FESVo and placed in the texture buffer at the computed position. As we are using a hashing algorithm to index the dataset, data retrieval is performed at a 


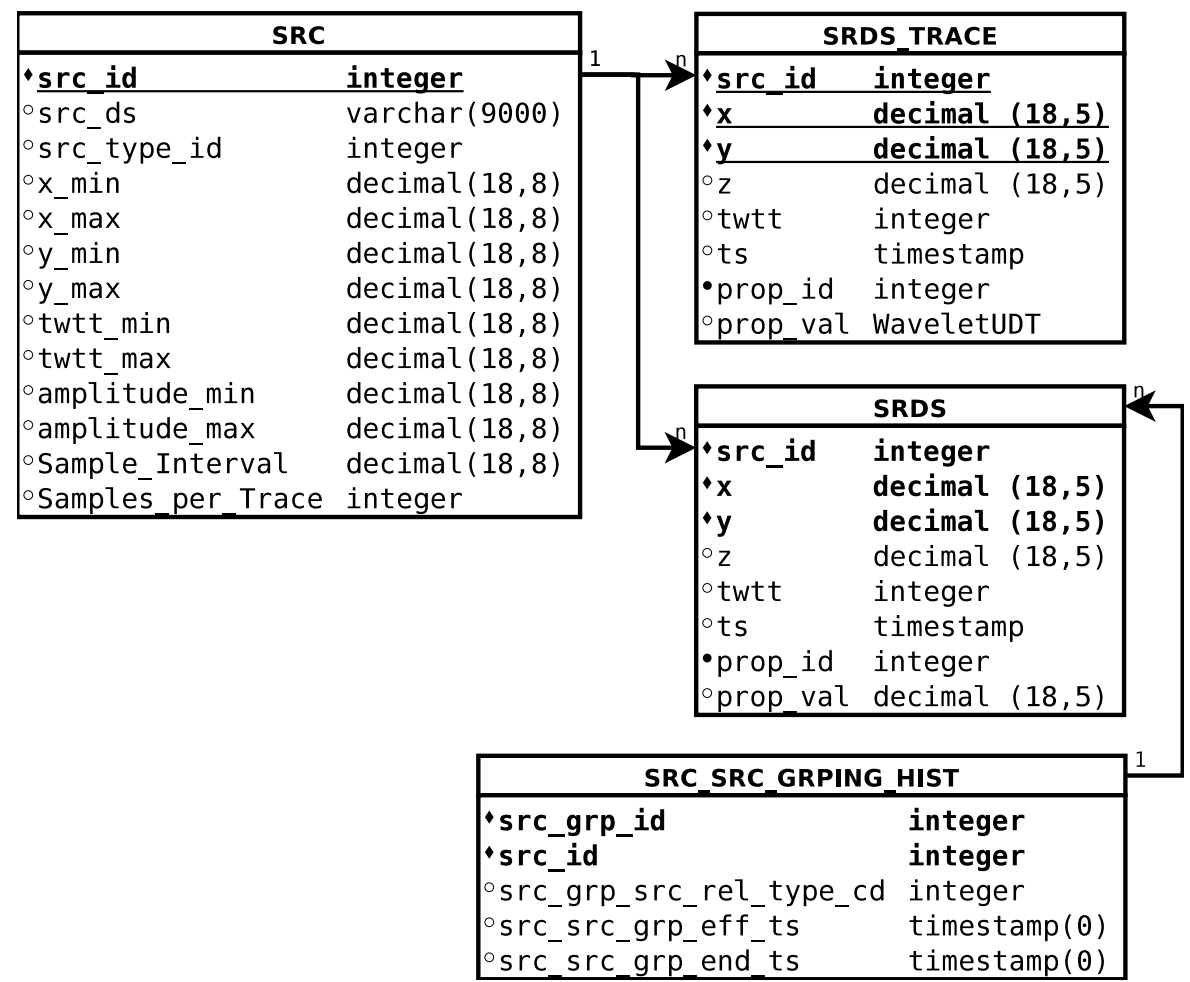

Figure 3: This diagram illustrates the extended SRDS database schema for geoscience. (twtt: two-way-travel time)

complexity of $O(k)$, where $\mathrm{k}$ is the number of data units being fetched from the database; this is independent of the total size of the table (dataset).

\subsubsection{Coordinate Mapping and Levels-of-Detail}

The architecture deals with three coordinate systems: (1) texture coordinate $(s, t, r)$-as in OpenGL, (2) local volume coordinate (localX, localY, localZ) per level-of-detail, and (3) global geographical coordinate $(x, y, z)$.

The mapping between coordinates takes place on the X-Y plane. At this stage of our work, no mapping is performed on the $z$-axis; a seismic trace is fully loaded to a $1 \mathrm{D}$ texture location $(s, t)$. This is because the trace length of raw seismic data is, usually, fixed across one dataset and relatively small, around 200 to 2000 samples per trace; each sample is a 4-byte floating point.

As illustrated in Figure 5, seismic data is not perfectly gridded but regularly distributed. Thus, we can divide the region into GIS cells of equal areas, each containing one trace. Each GIS cells corresponds to a $2 \mathrm{D}$ location in the local volume. This local volume now becomes the highest resolution level $(L O D O)$. Each subsequent level halves the dimension of its previous one; i.e. we rely on a decimationbased technique for downsampling. Since SRDS and, thus, the calculated GIS cells are regularly structured, a low resolution image can be obtained through direct downsampling; cells of higher levels-of-detail (lower resolution) are mapped to cells at $\angle O D O$ based on a regular decimation. Only one resolution version (the highest) of the dataset exists and real-time mapping is performed for lower resolution levels. The slight alteration as a result of the gridding (from a non-perfect grid in the real-world to a perfect grid in the local volume) is accepted by the industry.

\subsubsection{Data Lookup}

A GIS cell, in our seismic case, holds a subsurface dataset under a rectangular area (e.g. $12 \times 12$ meter square) of the real-world. In texture world, this is mapped to a single 1D dataset. To search inside a GIS cell in the database, we can choose between two modes: (1) general discovery mode and (2) specific cached mode. We maintain both modes and perform one depending on the task.

In the first mode, we have no knowledge in advance about the exact coordinates of the data units; thus it is a discovery mode. To query the database for a dataset which lies in a GIS cell, we explicitly query every possible location $(x, y)$ with a minimum step (e.g. 1 meter). The reason why explicit values of $x$ and $y$ are provided in the query is to perform 


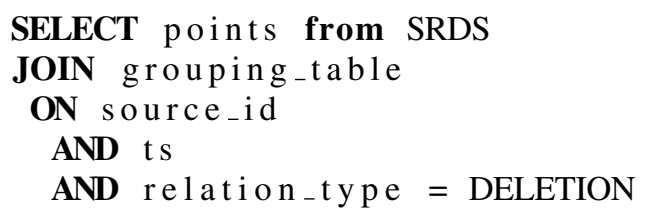

In this query, the baseline is the original interpretation which was first imported from an external source. We control the history tracking by manipulating the timestamp value.

\subsection{Rendering Engine}

At this stage, we adopt a back-to-front textured slice mapping rendering technique (Mcreynolds and Hui, 1997) along with a shader program, using OpenGL Shader Language (GLSL). Two texture objects (buffers) exist at any time: one for seismic raw data (volumetric datasets) and the other one is for all geological features.

\section{RESULTS AND CASE STUDIES}

In this section, we first present a case study performed by geoscientists from a university geoscience department. Then, we present some performance measures. For these we used Teradata DBMS, running virtually on a 64-bit Windows Server 2003. Both FESVo and the renderer engine were deployed on laptop and desktop machines equipped with graphics cards of $256 \mathrm{MB}$ to $1 \mathrm{~GB}$ of memory. Finally, we present and discuss a survey that we recently conducted on staff from the oil and gas industry as well as geoscientists from academia.

\subsection{Case Study on Geoscientists}

Six geoscientists from a university geoscience department participated in this case study. The participants were divided into three sessions. At each session, the participants were given an explication of the system and the purpose of the case study. Then each of the two participants in a session was given an access to the system, and were guided to load the same dataset at the same time from the centrally located database.

First, all participated geoscientists confirmed that the dataset was rendered correctly; their commercial software was considered as a guideline. We asked them to confirm this since the data in SRDS is completely restructured and thus this confirmation verifies our reconstruction (loading) method.

The participants were then asked to perform the following selected tasks using our system. For them, these cases are simple core tasks that may take part in their interpretation work flow. We selected these tasks only for the purpose of demonstrating the functionality of our architecture, such that provenance of users' interpretations is maintained using a two-way fine-grained visualization pipeline with a central relational database. In the following, we define a task then explain how it is technically achieved using our architecture.

\subsubsection{Task 1: Multi-User Horizon Time Shifting}

In this task, one user was asked to adjust a horizon by shifting its two-way-travel time (TWTT). Graphically, this time is the $z$ axis of an early-stage seismic imaging data; it is later converted into real depth. The process of time shifting a horizon can be done in several ways in respect to selecting where the shift is applied. In our implementation, we allow the user to select the following:

1. the horizon to which the shift is applied

2. a seed point

3. a shift value (+/-) (e.g. 50 milliseconds)

4. a diameter value to which the shifting is applied (e.g. 400 meters)

After setting these parameters, the time-shifting is implemented as follows. We start a deletion type grouping in SRDS linked to the original interpretation source ID and tagged with this user ID and a current timestamp. Then, all points lying within the selected diameter are inserted into the database in parallel threads, tagged with the user ID and the timestamp. Next, we end the deletion type grouping and start an insertion type grouping in SRDS with the same user ID but a new timestamp. Then, all points laying within the selected diameter are inserted into the database in parallel threads, with a new time value calculated in respect to the original value (this calculation is performed on the database) and tagged with the user ID and the new timestamp. Finally we end the grouping. Thus, user amendments are saved while the original interpretation is also maintained centrally with the original dataset. These steps are illustrated in Figure 6.

After the time-shift task was completed (took around 2-4 seconds), the second user in this session refreshed the application's view and was able to immediately visualize the changes on the horizon made by the first user. Figure 7 illustrates a similar view of this result.

In Figure 7, the original horizon, as shown in the left screenshot, consisted of 160,330 points (each is stored as a row in SRDS). The process of time-shift, 


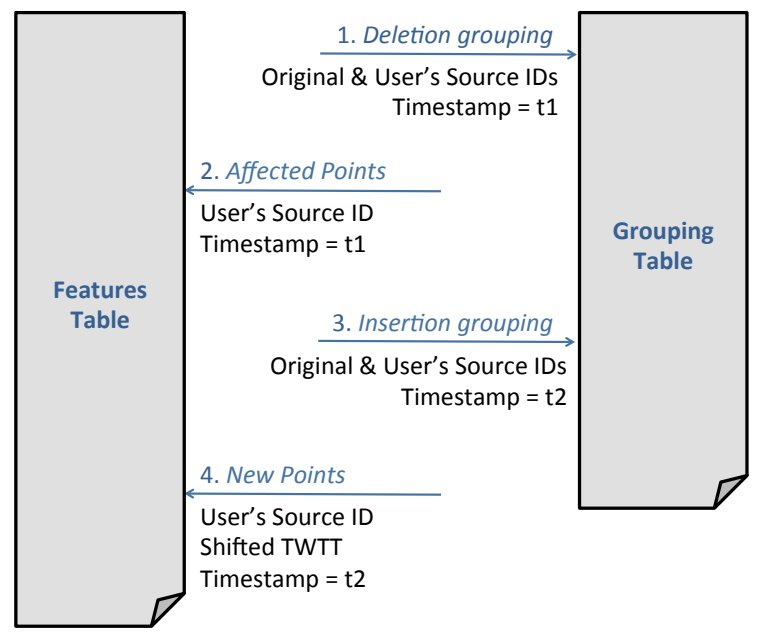

Figure 6: This figure shows the steps taken in interaction with the database for a user to shift a previously interpreted horizon.
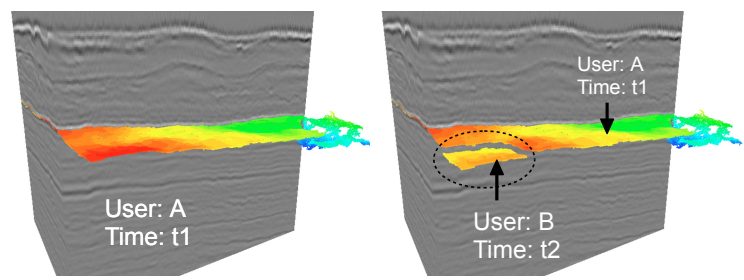

Figure 7: The screenshot on the left shows an interpreted horizon by user $\mathrm{A}$ at time $\mathrm{t} 1$. The screenshot on the right shows a partially shifted horizon by user B at time $\mathrm{t} 2$. The change (shift) takes place only on the affect points since we implemented a fine-grained provenance.

as shown in the right screenshot, resulted in 1,560 points tagged as deleted (from the original interpretation) and the same number of points $(1,560)$ tagged as inserted (forms the shifted area). Thus, a total number of 163,450 points form this horizon including its provenance and new changes; this is a result of a finegrained granularity model. In comparison to a coarsegrained (file-based) granularity, any small change to a feature object means a new whole object if we need to maintain its provenance. Thus, we end up with around double the number of original points, 320,660 points.

\subsubsection{Task 2: Deletion of an Interpreted Object with History Tracking}

In this task, we assume that two users have previously added to an existing interpretation of a horizon from a particular source. A senior (more expert) user later visualized both interpretations and decided that one is more accurate than the other and therefore wanted to delete the less accurate interpretation.

One user of a session (acted as an expert) was asked to select a session with a data insertion tag to delete. Technically, this is achieved as follows. As in Task 1, we start a deletion type grouping in SRDS tagged with this user ID and a current timestamp. Then, a single update query containing the user ID and timestamp of the session to be deleted is executed. This results in re-inserting the points of this session but tagged with the expert user's ID and a timestamp of the created deletion type grouping. We then end this grouping. We then refresh FESVo and reload the latest version of interpretations which includes the original (previously existed) version and the additional interpretation by the more accurate user.

As we record a timestamp when starting a grouping between different interpretation sources, we can go back in history to visualize earlier versions. In this task, the second user of this session was able to track the history of this horizon; this is illustrated in Figure 8.

\subsection{Performance Measure}

Our current aim is to achieve data provenance with an acceptable performance. Our tests were performed on laptop equipped with a graphics cards of $256 \mathrm{MB}$ of memory. The database was running virtually on a single-node 64-bit Windows Server 2003. The total size of the tables, which mainly include three seismic imaging datasets and several users' interpretations, were around $35 \mathrm{~GB}$.

Over a local area network (LAN), we were able to initially load the seismic traces at a level of detail with a lower resolution of one seismic volume in around 7 to 8 seconds; this level had a size of $38 \mathrm{MB}$. The feature object (horizon) was loaded in around 1 to 2 second(s); both traces and the feature object forms the output is illustrated in Figures 7 or 8. The loading process used 4 threads concurrently. Each SQL command is a multi-statement request consists of a maximum of 16 point-to-point queries. Each query is a fetch request of a macro previously set up to query a single trace or a set of feature points against a unique geographical location $(x, y)$.

By experiment, we found that the number of threads to run concurrently is preferred to be multiple of the database's parallel units, known by Teradata as Access Module Processor (AMP) (Ballinger, 2009). The database used in our tests has four AMPs. Thus, as shown on the graph of Figure 9, the throughput becomes stable when using four threads or more. This would only be the case when performing the queries on a fast connection, as is the case with the test of Figure 9. When using a slower connection, more threads, up to a limit, would boosts the overall throughput as the number of threads overcomes the slowness in con- 

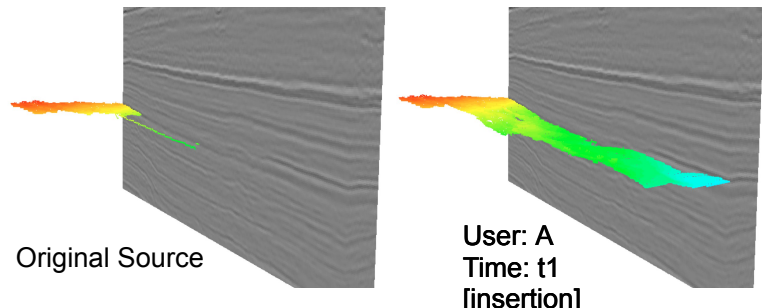

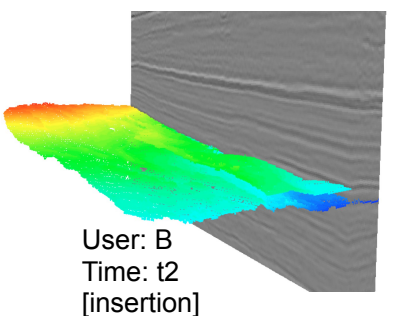

[insertion]

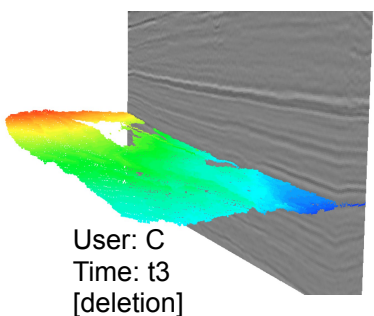

[deletion]

Figure 8: From left, the second screenshot shows some interpretation added to the original one (first one from left) by User $\mathrm{A}$ at time $\mathrm{t} 1$. The third screenshot shows more contribution by User B at time $\mathrm{t} 2$. The fourth screenshot, an expert user (User C) decided to delete the interpretation by User A due to, for example, lack of accuracy. The interpretation of User A is in fact not deleted but tagged as deleted. Users, therefore, can go back in history and visualize previous versions of interpretations.

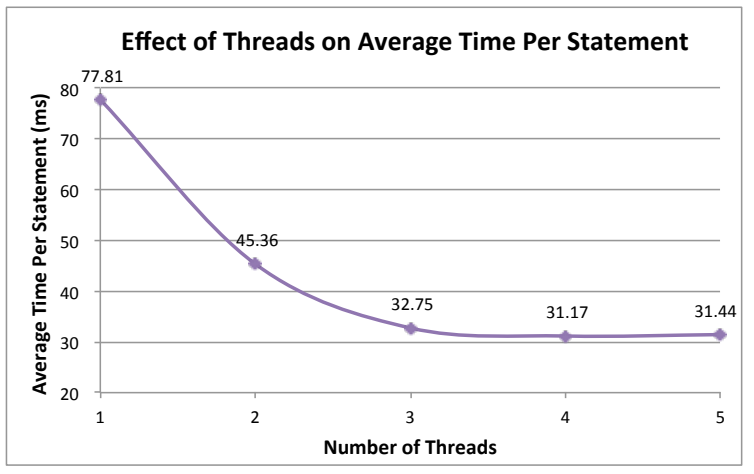

Figure 9: The number of threads to run concurrently is preferred to be multiple of the database's parallel modules. Using a high speed connection and a database with 4 parallel modules, the throughput becomes stable after 4 concurrent threads.

nections.

\subsection{Survey}

We recently made a non-public survey of 18 senior staff from oil and gas companies (5 geoscientists and 13 IT staff who explicitly support and maintain subsurface data, hardware, and software infrastructures) and 6 geoscientists from a university (5 postgraduate students and 1 senior staff). The purpose of the survey was to understand the following:

1. the importance of a collaborative environment and data provenance for seismic interpretation,

2. the differences between a seismic interpretation environment in universities and in the industry,

3. the challenges IT staff encountered to fulfil the need of seismic visualization and interpretation in an efficient data management manner.

The collaboration in this context refers to the ability of users to share their results of interpretations and work together to produce such results. Ten out of eleven of the participated geoscientists perceived that a collaborative visualization and interpretation environment is "very important". The participants from the industry added that collaboration "raises productivity." We observed that the need of a collaboration was more obvious to geoscientists in industry than in universities where the work is almost performed individually. The same ratio also perceived that it is overall challenging to collaborate on seismic interpretation using existing software. The geoscientists from the industry highlighted this challenge on sharing their interpretations with other teams; for example between the interpreters and engineers. In such an industry, it is often that each team uses different software, and each software uses its proprietary internal format. All participated geoscientists perceived that data provenance, including history tracking, is important in seismic interpretation; most of the participants from the industry added that history tracking "raises efficiency." Looking into the challenge of history tracking, geoscientists from the universities did not find this challenging while the industry saw that it is "manageable for recent interpretations but a bit challenging for very old datasets," this perhaps clarifies the former finding.

The response from the IT staff highlighted the technical challenges and thus area of improvements, where geoscientists might not perceive this in the same way, since it is the job of the IT staff to do this for them. Ten out of thirteen IT staff perceived that users cannot immediately access all subsurface data for visualization or interpretation in a high availability fashion; but data access needs some initial preparation. They share with the geoscientists the perception of challenging collaboration; $46 \%$ found that sharing users' results with any other staff members is a time consuming task as it requires to export then import data to be shared. The participants suggested that to enhance the infrastructure, for a collaboration that maximizes productivity, the industry need to introduce a "more integrated data environment", "use cloud-based technology", "standardize the workflow 
between all users", and "have shared databases among all disciplines (teams)." In addition, they evaluated the move of data between application as it negatively affect productivity; $62 \%$ of them emphasised this as a "high" effect and it "needs a great attention." Eleven out of thirteen believed that centralizing subsurface datasets would improve data management, but this "has been challenging up to now." Some of their suggested reasons for not centralizing datasets were; the proprietary internal format of multiple applications and the lack of a well integrated environment in the industry. The need of a "standard data repository" was highlighted.

\section{CONCLUSION AND FUTURE WORK}

In this paper we have demonstrated a proof of concept of our data-centric approach to fine-grained data provenance of users' visual interpretations, applying this to seismic imaging data. Our method represents and accumulates users' interpretations of geological features as fine-grained metadata and combines it with the raw seismic data into a single storage. Currently we use a relational database; we intend to test our method with other forms of data storage. We link this to a renderer through a loading mechanism and also allow users' amendments of interpretations to flow back as new metadata to the data storage. In this paper, we have presented a case study on geologists testing our architecture and some performance results. We have also presented a survey which shows a need for an efficient data management in seismic visualization; we believe that our architecture is a step into addressing this need.

Our plan for the future is to integrate our architecture with feature extraction techniques and algorithms, such as the work presented by Höllt et. al. (Höllt et al., 2011). Also, we plan to provide more interactive functionalities available to users' interpretations of features. In addition, it is vital to test our methods on massive datasets due to the nature and demands of the oil and gas industry. We plan to scale our implementation into massive parallel processing databases to support massive datasets at a high performance. Finally, in this paper we have considered only seismic imaging datasets. However, our method can potentially be extended to support other types of spatial data, for example from oceanography, space physics, and medical imaging.

\section{REFERENCES}

Ahrens, J., Brislawn, K., Martin, K., Geveci, B., Law, C., and Papka, M. (2001). Large-scale data visualization using parallel data streaming. IEEE Computer Graphics and Applications, 21(4):34-41.

Al-Naser, A., Rasheed, M., Brooke, J., and Irving, D. (2011). Enabling Visualization of Massive Datasets Through MPP Database Architecture. In Carr, H. and Grimstead, I., editors, Theory and Practice of Computer Graphics, pages 109-112. Eurographics Association.

Al-Naser, A., Rasheed, M., Irving, D., and Brooke, J. (2013a). A Data Centric Approach to Data Provenance in Seismic Imaging Data. In 75th EAGE Conference \& Exhibition incorporating SPE EUROPEC, London. EAGE Publications bv.

Al-Naser, A., Rasheed, M., Irving, D., and Brooke, J. (2013b). A Visualization Architecture for Collaborative Analytical and Data Provenance Activities. In Information Visualisation (IV), 2013 17th International Conference on, pages 253-262.

Alvarez, F., Dineen, P., and Nimbalkar, M. (2013). The Studio Environment: Driving Productivity for the E\&P Workforce. White paper, Schlumberger.

Bacon, M., Simm, R., and Redshaw, T. (2003). 3-D Seismic Interpretation. Cambridge University Press.

Ballinger, C. (2009). The Teradata Scalability Story. Technical report, Teradata Corporation.

Bavoil, L., Callahan, S., Crossno, P., Freire, J., Scheidegger, C., Silva, C., and Vo, H. (2005). VisTrails: Enabling Interactive Multiple-View Visualizations. In Visualization, 2005. VIS 05. IEEE, pages 135-142. IEEE.

Biddiscombe, J., Geveci, B., Martin, K., Moreland, K., and Thompson, D. (2007). Time dependent processing in a parallel pipeline architecture. IEEE Transactions on Visualization and Computer Graphics, 13(6):13761383.

BP (2012a). BP Energy Outlook 2030. Technical report, London.

BP (2012b). BP Statistical Review of World Energy June 2012. Technical report.

Brooke, J. M., Marsh, J., Pettifer, S., and Sastry, L. S. (2007). The importance of locality in the visualization of large datasets. Concurrency and Computation: Practice and Experience, 19(2):195-205.

Castanie, L., Levy, B., and Bosquet, F. (2005). VolumeExplorer: Roaming Large Volumes to Couple Visualization and Data Processing for Oil and Gas Exploration. In IEEE Visualization, volume im, pages 247 254. Ieee.

Gosink, L. J., Anderson, J. C., Bethel, E. W., and Joy, K. I. (2008). Query-driven visualization of time-varying adaptive mesh refinement data. IEEE transactions on visualization and computer graphics, 14(6):1715 -1722 .

Hadwiger, M., Ljung, P., Salama, C. R., and Ropinski, T. (2009). Advanced Illumination Techniques for GPU Volume Raycasting. In ACM SIGGRAPH Courses Program, pages 1-166, New York, NY, USA. ACM. 
Halliburton-Landmark (2013). GeoProbe Volume Visualization. https://www. landmarksoftware.com/ Pages/GeoProbe. aspx.

Hawtin, S. and Lecore, D. (2011). The business value case for data management - a study. Technical report, CDA $\&$ Schlumberger.

Höllt, T., Beyer, J., Gschwantner, F., Muigg, P., Doleisch, H., Heinemann, G., and Hadwiger, M. (2011). Interactive seismic interpretation with piecewise global energy minimization. In Pacific Visualization Symposium (PacificVis), 2011 IEEE, pages 59-66, Hong Kong.

Ikeda, R. and Widom, J. (2009). Data Lineage: A Survey. Technical report, Stanford University.

Jomier, J., Aylward, S. R., Marion, C., Lee, J., and Styner, M. (2009). A digital archiving system and distributed server-side processing of large datasets. In Siddiqui, K. M. and Liu, B. J., editors, Proc. SPIE 7264, Medical Imaging 2009: Advanced PACS-based Imaging Informatics and Therapeutic Applications, volume 7264, pages 726413-726413-8.

Jomier, J., Jourdain, S., and Marion, C. (2011). Remote Visualization of Large Datasets with MIDAS and ParaViewWeb. In Proceedings of the 16th International Conference on 3D Web Technology, pages 147-150, Paris, France. ACM.

Karvounarakis, G., Ives, Z. G., and Tannen, V. (2010). Querying data provenance. In Proceedings of the 2010 ACM SIGMOD International Conference on Management of data, SIGMOD '10, pages 951-962, New York, NY, USA. ACM.

Lin, J. C.-R. and Hall, C. (2007). Multiple oil and gas volumetric data visualization with GPU programming. Proceedings of SPIE, 6495:64950U-64950U-8.

Ma, C. and Rokne, J. (2004). 3D Seismic Volume Visualization, volume VI, chapter 13, pages 241-262. Springer Netherlands, Norwell, MA, USA.

Mcreynolds, T. and Hui, S. (1997). Volume Visualization with Texture, chapter 13, pages 144-153. SIGGRAPH.

Moreland, K. (2013). A Survey of Visualization Pipelines. Visualization and Computer Graphics, IEEE Transactions on, 19(3):367-378.

Patel, D., Bruckner, S., and Viola, I. (2010). Seismic volume visualization for horizon extraction. In Proceedings of IEEE Pacific Visualization, volume Vi, pages 73-80, Taipei, Taiwan. IEEE.

Patel, D., Sture, O. y., Hauser, H., Giertsen, C., and Eduard Gröller, M. (2009). Knowledge-assisted visualization of seismic data. Computers \& Graphics, 33(5):585596.

Pavlo, A., Paulson, E., Rasin, A., Abadi, D. J., DeWitt, D. J., Madden, S., and Stonebraker, M. (2009). A comparison of approaches to large-scale data analysis. In Proceedings of the 2009 ACM SIGMOD International Conference on Management of data, pages 165-178. ACM.

Plate, J., Holtkaemper, T., and Froehlich, B. (2007). A flexible multi-volume shader framework for arbitrarily intersecting multi-resolution datasets. IEEE transactions on visualization and computer graphics, 13(6):1584-91.

Plate, J., Tirtasana, M., Carmona, R., and Fröhlich, B. (2002). Octreemizer: a hierarchical approach for interactive roaming through very large volumes. In Data Visualisation, pages 53-60. Eurographics Association.

Rahimi, S. K. and Haug, F. S. (2010). Query Optimization. Wiley.

Robein, E. (2010). Seismic Imaging: A Review of the Techniques, their Principles, Merits and Limitations. EAGE Publications bv.

Scheidegger, C. E., Vo, H., Koop, D., Freire, J., and Silva, C. T. (2007). Querying and creating visualizations by analogy. IEEE transactions on Visualization and Computer Graphics, 13(6):1560-1567.

Schlumberger (2013). Petrel Seismic to Simulation Software. http://www.slb.com/services/software/ geo/petrel.aspx.

Simmhan, Y. L., Plale, B., and Gannon, D. (2005). A survey of data provenance in e-science. SIGMOD Rec., 34(3):31-36.

Society of Exploration Geophysicists (2002). SEG Y rev 1 Data Exchange Format. Technical Report May.

Stockinger, K., Shalf, J., Wu, K., and Bethel, E. (2005). Query-Driven Visualization of Large Data Sets. In Visualization, 2005. VIS 05. IEEE, pages 167-174. IEEE.

Visualization Sciences Group (2013). Avizo Earth. http: //www.vsg3d.com/avizo/earth.

Vo, H., Bronson, J., Summa, B., Comba, J., Freire, J., Howe, B., Pascucci, V., and Silva, C. (2011). Parallel Visualization on Large Clusters using Map Reduce. In Large Data Analysis and Visualization (LDAV), 2011 IEEE Symposium on, pages 81-88. IEEE.

$\mathrm{Wu}, \mathrm{K}$. (2005). FastBit: an efficient indexing technology for accelerating data-intensive science. Journal of Physics: Conference Series, 16:556-560.

Wu, K., Ahern, S., Bethel, E. W., Chen, J., Childs, H., Cormier-Michel, E., Geddes, C., Gu, J., Hagen, H., Hamann, B., Koegler, W., Lauret, J., Meredith, J., Messmer, P., Otoo, E., Perevoztchikov, V., Poskanzer, A., Prabhat, Rübel, O., Shoshani, A., Sim, A., Stockinger, K., Weber, G., and Zhang, W.-M. (2009). FastBit: interactively searching massive data. Journal of Physics: Conference Series, 180:012053.

Zhang, S. and Zhao, J. (2010). Feature Aware Multiresolution Animation Models Generation. Journal of Multimedia, 5(6):622-628. 